\title{
Epidemiologia da hipovitaminose D em gestantes no Brasil: uma revisão sistemática
}

\section{Epidemiology of hypovitaminosis $D$ in Brazil: a sistematic review}

Fabiana Cândida de Queiroz Santos Anjos', Edison Benedito da Luz Brito Junior², Thiago Delmondes Feitosa², Marcello Levigne Silva Araújo², Renata Junqueira Pereira ${ }^{3}$

\section{RESUMO}

A hipovitaminose $D$ é um problema de saúde pública e as gestantes se enquadram em um grupo de risco, visto que a deficiência de vitamina D (VD) está envolvida na fisiopatologia de várias intercorrências gestacionais, como pré-eclâmpsia e diabetes gestacional. Estudos apresentam prevalências de hipovitaminose D em gestantes entre $18 \%$ e $84 \%$, conforme hábitos locais de vestuário e local de residência dos indivíduos. Esta revisão teve como objetivo investigar a epidemiologia da hipovitaminose D entre gestantes, por região do Brasil. Realizaram-se buscas nas bases de dados MEDLINE -via PubMed, LILACS e SCIELO, com os descritores: "Vitamina D", "Gravidez/Gestação" e "Brasil" (em português e inglês). Foram encontrados 267 artigos que, após aplicação dos critérios de inclusão e exclusão, se resumiram a 4 artigos. RESULTADOS: O estudo realizado no Rio de Janeiro com 199 pacientes $(29,6 \%$ suficientes), enquanto que em Curitiba tiveram 520 gestantes (19,2\% suficiente). O estudo realizado na Bahia com 190 gestantes (44,2\% suficiente) e o de São Bernardo do Campos com 144 gestantes (34,5\% suficiente). A média de suficiência no Brasil foi de $26,9 \%$. Conclui-se que existe uma grande variedade entre uma região e outra do país na hipovitaminose $D$, mais significativa quando mais distante do Equador.

Palavras-chave: Vitamina D. Gestação. Brasil

\section{ABSTRACT}

Hypovitaminosis $D$ is a public health issue and pregnant women belong to a group at risk, since the vitamin $\mathrm{D}$ deficiency is involved in the pathophysiology of various gestational diseases, such as preeclampsia, and gestational diabetes. Studies have shown a variation between $18 \%$ and $84 \%$ in the prevalence of this deficiency among pregnant women, based on local clothing habits and place of residence. This review aims to evaluate the epidemiology of hypovitaminosis $D$ in pregnant women in different regions of Brazil. The research was performed at online databases (MEDLINE-via PubMed, LILACS and SCIELO), with the keywords: "Vitamin D", "Pregnancy/Gestation" and "Brazil" (in Portuguese and English). There were 267 articles found, which after application of the inclusion and exclusion criteria, 4 articles remained. RESULTS: The study conducted in Rio de Janeiro had 199 patients (29.6\% sufficient). In Curitiba with 520 pregnant women (19.2\% sufficient). In Bahia with 190 pregnant women (44.2\% sufficient). In São Bernardo do Campos with 144 pregnant women (34.5\% sufficient). The average sufficiency in Brazil was $26.9 \%$. The findings conclude that there is a great variety between regions of the country in regards of hypovitaminosis $D$, more significant when further away from the equator line.
Mestranda em Ciências da Saúde. Universidade Federal do Tocantins.

E-mail: fcqsa@uol.com.br

2 Acadêmico do curso de medicina da Universidade de Gurupi.

3 Doutora Universidade Federal do Tocantins. 


\section{INTRODUÇAOO}

Destacando-se como um problema de saúde pública mundial (MAEDA et al., 2014), a deficiência ou insuficiência de vitamina $D(V D)$ tem enquadrado as gestantes em um grupo de alto risco, que deve ser acompanhado e estudado (MULLIGAN et al., 2010). Variações nas composições dos polivitamínicos comumente prescritos na gestação, ingestões alimentares inadequadas, questões ambientais (exposição ao sol) e de estilos de vida diferentes são responsáveis pelas elevadas prevalências de hipovitaminose VD, tanto em países em desenvolvimento, como nos desenvolvidos (DAWODU; WAGNER, 2012; HOSSEIN-NEZHADA; HOLICK, 2013; NARCHI et al., 2010). Os estudos mostram prevalências de hipovitaminose D, variando entre 18 e $84 \%$ em gestantes, conforme os hábitos locais de vestuário e locais de residência/país (DAWODU; WAGNER, 2012; JAIN et al., 2011).

A literatura afirma que a 25 hidroxi-vitamina D é o marcador sanguíneo de escolha para avaliar os níveis séricos e o estado nutricional de vitamina D (HOLICK, 2007; NORMAN; BOUILLON, 2010; HOLICK et al., 2011; HEANEY, 2013), classificando os indivíduos como deficientes, quando apresentam concentrações abaixo de $50 \mathrm{nmol} / \mathrm{L}$; e como insuficientes, quando os valores encontrarem-se entre 50 e $75 \mathrm{nmol} / \mathrm{L}$. Dessa forma, indivíduos com níveis séricos de 25 hidroxi-vitamina $D$ inferiores a $75 \mathrm{nmol} / \mathrm{L}$ são considerados com hipovitaminose D (HOLICK, 2007; HOLICK et al., 2012).

Nos indivíduos adultos a produção cutânea é a fonte principal de calciferol, via exposição solar, e somente $10 \%$ das reservas corporais provêm dos hábitos alimentares (DAWODU; WAGNER, 2012). Recomendar a exposição solar ao paciente torna-se algo bastante impreciso, visto que irá depender do grau de instrução do paciente, do uso de protetor solar, da latitude e altitude onde ele reside, do período do dia em que a exposição ocorre, da quantidade de melanina em cada indivíduo e da quantidade de radiação (Ultravioleta B) UVB presente (HOSSEIN-NEZHAD; HOLICK, 2013). Quanto à exposição à radiação UVB, verifica-se que a síntese e a absorção cutânea de VD somente ocorrem na presença dessa radiação, que atinge seu máximo na irradiação solar entre 10 e 15 horas, no Equador, ou em locais próximos a ele. Nesse sentido, a recomendação é expor os membros superiores e inferiores nesse horário, durante 5 a 30 minutos, pelo menos duas vezes por semana (HOLICK, 2007).

Torna-se essencial considerar que, toda e qualquer situação ou condição que estreite a exposição ao sol, poderá predispor o paciente à deficiência de vitamina $D$, 
acrescentando-se o uso de fotoproteção (REICHRATH; NURNBERG, 2009) e a utilização de vestuário religioso, como batinas, véus, burca, paramentos (GANNAGE-YARED et al., 2000; JAMALI et al., 2013).

As principais fontes alimentares de vitamina D são os óleos (principalmente o óleo de fígado de bacalhau), os peixes ricos em gorduras (como salmão, cavala, atum), cogumelos frescos e secos ao sol, gema de ovo, sardinha (LIPS, 2001; HOLICK, 2007; NORMAN; BOUILON, 2010; WACKER; HOLICK, 2013) e produtos enriquecidos (cereais, iogurtes, leite e margarina) (BASILE, 2014). A depender da fonte nutricional, haverá prevalência de um determinado tipo de vitamina D; nos alimentos de origem vegetal encontra-se a forma de ergocalciferol (D2), já nos de origem animal é encontrada na forma de colecalciferol (D3), ambas se diferenciam somente por uma ligação dupla extra e um grupo metil na cadeira longa lateral de D2 (TRIPKOVIC et al., 2012).

Durante o período gestacional as necessidades nutricionais tornam-se elevadas, devido às alterações fisiológicas da gravidez e às demandas fetais, que impõem à mulher profundas mudanças psicológicas, endócrinas ou somáticas (BASILE, 2014). A hipovitaminose D na gestante pode levar ao baixo peso ao nascer, diabetes gestacional, vaginose bacteriana e pré-eclampsia, conforme metanálise de Aghajafari et al. (2013); além de restrição do crescimento intrauterino e parto pré-termo (ASEMI et al., 2013). Observa-se ainda interferências da VD nos processos de invasão trofoblástica, nos níveis pressóricos, na proteinúria e na imunomodulação, auxiliando no controle das Doenças Hipertensivas Específicas da Gestação (BENACHI et al., 2013).

Sobre a orientação para a suplementação de VD é preconizado um valor de 600 Ul/dia para grávidas, segundo a Sociedade Brasileira de Endocrinologia e Metabologia (SBEM) (MAEDA et al., 2014). Pacientes morenos ou negros possuem menor síntese de VD, uma vez que a melanina dificulta a biotransformação cutânea da molécula de 7hidrocolesterol em VD. Assim, esses pacientes necessitam de 10 a 50 vezes mais tempo de exposição solar, afim de produzirem níveis de VD semelhantes ao que ocorre para os indivíduos de pele clara (DOWSON-HUGHES; HARRIS; DALLAL, 1997).

A placenta responde mediante as concentrações de VD, funcionando como moduladora de implantação na síntese de citocinas e na resposta imune à infecção (BASILE, 2014). Por via placentária, a 25-hidroxivitamina D é transferida da mãe para o feto, principalmente durante o terceiro trimestre, mostrando que dosagens maternas refletem níveis fetais (MA et al., 2010). Além disso, existem ainda receptores placentários de VD, que metabolizam a forma ativa da 25-hidroxivitamina D, elevando assim a 
disponibilidade fetal (DAWODU; AKINBI, 2013). Portanto, a via transplacentária representa a fonte primária de VD ao recém-nascido, durando os estoques adquiridos da mãe até a oitava semana após o nascimento (DAWODU; AKINBI, 2013).

Garantir concentrações ideais de VD para saúde da gestante e do feto é fundamental, independentemente do trimestre gestacional (BARTOSZEWICZ et al., 2013). Atualmente, a dosagem de rotina da 25 hidroxi-vitamina $D$ não faz parte do calendário mínimo de exames pré-natais, proposto pelo Ministério da Saúde; nem tampouco, a suplementação de VD figura dentre os programas nacionais de suplementação de micronutrientes. Há que se considerar ainda que o Brasil, em função de sua grande dimensão territorial, possui climas variados (variando a exposição solar), bem como as diferentes regiões possuem hábitos alimentares diversificados, sendo provável que as prevalências de hipovitaminose $D$ variem também entre as regiões brasileiras.

No Brasil existem poucos estudos sobre a epidemiologia da hipovitaminose D em gestantes, dessa forma, essa revisão teve como objetivo levantar e descrever os estudos epidemiológicos sobre a hipovitaminose $\mathrm{D}$ em gestantes, nas diferentes regiões do Brasil.

\section{METODOS}

Trata-se de um estudo de revisão literária sistemática, seguindo a metodologia proposta por Preferred Reporting Items for Systematic Reviews and Meta-Analyses (PRISMA), baseada em pesquisa on-line nas bases de dados Medical Literature Analysis and Retrieval System Online /PubMed (Medline), Literatura Latino-Americana e do Caribe em Ciências da Saúde (LILACS) e Scientific Electronic Library Online (SciELO), utilizando os termos: "Vitamina D", "Gravidez/Gestação" e "Brasil”, nos idiomas português e inglês. Nesta revisão incluíram-se apenas artigos originais, independentemente do idioma em que foram escritos e não se considerou recorte temporal.

A partir das combinações dos descritores, identificaram-se 176 artigos completos em inglês e 91 artigos em português, perfazendo um total de 267 artigos relacionados ao tema. Após a leitura dos títulos foram excluídos os repetidos, restando 84 artigos e, após análise dos resumos foram excluídos os que tratavam de outros micronutrientes como ferro, ácido fólico e outras vitaminas, não mencionando a VD. Também foram excluídos os que estudavam a vitamina $\mathrm{D}$, porém abordando temáticas como complicações maternas e fetais. Foram incluídos apenas os estudos que focaram na epidemiologia da 
hipovitaminose $D$, na gestação e na população brasileira. Um total de 4 artigos foi selecionado para leitura na íntegra.

Os dados publicados na literatura foram categorizados conforme os pontos de corte: vitamina D deficiente $(<50 \mathrm{nmol} / \mathrm{L})$, insuficiente $(\geq 50$ e $<75 \mathrm{nmol} / \mathrm{L})$ e suficiente $(\geq$ $75 \mathrm{nmol} / \mathrm{L})$, de acordo com o preconizado pelo Endocrine Society Practice Guidelines (HOLICK et al., 2012).

As categorias de estado nutricional de vitamina $D$, nas diferentes regiões e suas latitudes foram comparados e analisados utilizando-se o teste de Qui-Quadrado, no software Statistical Package for Social Sciences (SPSS), versão 25.0.

\section{RESULTADOS}

No estudo de Figueiredo et al. (2017) foram acompanhadas 199 gestantes saudáveis, na cidade do Rio de Janeiro (localizada na Latitude: $22^{\circ} 54^{\prime} 13^{\prime \prime}$ Sul), com dosagem da 25 hidroxi-vitamina $D$ nos 3 trimestres de gestação e 178 gestantes com dosagem da 1,25 dihidroxi-vitamina D.

Encontou-se uma maior prevalência de deficiência de vitamina $D$ entre as gestantes que ingressaram no estudo no inverno e na primavera, em comparação com aquelas que ingressaram no verão ou no outono. A prevalência de gestantes com concentrações plasmáticas de 25 hidroxi-vitamina D inferiores a $75 \mathrm{nmol} / \mathrm{L}$ foi de 70,4\% no primeiro trimestre de gestação; 41,0\%, no segundo e 33,9\%, no terceiro. A prevalência de gestantes com concentrações plasmáticas de 25 hidroxi-vitamina $D$ inferiores a 50 $\mathrm{nmol} / \mathrm{L}$ foi de $16,1 \%$ no primeiro trimestre gestacional; $11,2 \%$, no segundo e $10,2 \%$, no terceiro. (FIGUEIREDO et al., 2017).

No presente estudo, para comparar com os demais trabalhos utilizou-se apenas os dados da primeira dosagem, ou seja o primeiro trimestre. Encontrando $16,1 \%$ de deficiência, 54,3\% de insuficiencia e $29,6 \%$ de suficiente.

Chrisostomo et al. (2018) realizaram estudo transversal, com pacientes de consultas pré-natais regulares, do Departamento de Obstetrícia de dois hospitais universitários de Curitiba, Paraná (Latitude: 2525' 42" Sul). Foram estudadas 520 gestantes, em diferentes idades gestacionais. Durante o estudo 264 gestantes foram avaliadas no inverno e 256 no verão. Os níveis de vitamina $D$, nos períodos do inverno e verão variaram de $11,23 \mathrm{nmol} / \mathrm{L}$ a $141,27 \mathrm{nmol} / \mathrm{L}$ com um valor mediano de 53,41 nmol/L (intervalo interquartil de 39,94 - 69,89 nmol/L). Usando o mesmo ponto de corte preconizado pela Endocrine Society Practice Guidelines (HOLICK et al., 2012), foi 
observado um total de 227 (43,7\%) gestantes com deficiência de vitamina D; 193 (37,1\%) com insuficiência e 100 (19,2\%) estavam com níveis sanguíneos normais de vitamina D.

Pereira-Santos et al.(2018) em um estudo transversal na cidade de Santo Antônio de Jesus, Bahia, Brasil, (latitude 12 57'12" Sul), com pacientes do Sistema Único de Saúde (SUS), estudaram gestantes saudáveis, com idade gestacional inferior a 32 semanas, nos períodos de inverno e verão. Foram estudadas 190 gestantes, na faixa etária entre 18-29 anos (63\%), de baixa renda (64\%). Do total de gestantes estudadas, 27 $(14,2 \%)$ apresentaram deficiência de Vitamina D, 79 (41,7\%) tinham insuficiência de vitamina D e $84(44,2 \%)$ apresentaram níveis normais da vitamina.

Kassai et al.(2018) realizaram estudo transversal em São Bernardo do Campo, São Paulo, (latitude 2341'40" Sul), com 66 mulheres que deram à luz bebês pré-termo (IG <32 semanas) e 92 mulheres com partos a termo e realizaram a dosagem de 25hidroxi-vitamina D em 144 das gestantes, sendo observado 34,8\% de deficiência, $37,5 \%$ de insuficiência e $34,5 \%$ com níveis adequados da vitamina.

Tabela 1: Estado Nutricional de Vitamina D em gestantes estudadas em diferentes regiões do Brasil.

\begin{tabular}{|c|c|c|c|c|c|c|c|}
\hline \multirow[t]{2}{*}{ AUTOR } & \multirow[t]{2}{*}{ ANO } & \multirow[t]{2}{*}{ REGIÃO } & \multirow[t]{2}{*}{ LATITUDE } & \multirow[t]{2}{*}{$\mathbf{N}$} & \multicolumn{3}{|c|}{ ESTADO NUTRICIONAL DE VITAMINA D } \\
\hline & & & & & Deficiente & Insuficiente & Adequado \\
\hline Figueiredo et al. & 2017 & $\begin{array}{l}\text { Rio de } \\
\text { Janeiro, RJ }\end{array}$ & $\begin{array}{c}22^{\circ} 54^{\prime} 13^{\prime \prime} \\
\text { Sul }\end{array}$ & 199 & $\begin{array}{c}32 \\
(16,1 \%)\end{array}$ & $\begin{array}{c}108 \\
(54,3 \%)\end{array}$ & $\begin{array}{c}59 \\
(29,6 \%)\end{array}$ \\
\hline Chrisostomo et al. & 2016 & Curitiba, PR & $25^{\circ} 25^{\prime} 42^{\prime \prime}$ & 520 & $\begin{array}{c}227 \\
(43,7 \%)\end{array}$ & $\begin{array}{c}193 \\
(37,1 \%)\end{array}$ & $\begin{array}{c}100 \\
(19,2 \%)\end{array}$ \\
\hline $\begin{array}{l}\text { Pereira-Santos et } \\
\text { al. }\end{array}$ & 2017 & $\begin{array}{l}\text { Santo } \\
\text { Antônio de } \\
\text { Jesus, BA }\end{array}$ & $\begin{array}{c}12^{\circ} 57^{\prime} 12 " ' \\
\text { Sul }\end{array}$ & 190 & $\begin{array}{c}27 \\
(14,2 \%)\end{array}$ & $\begin{array}{c}79 \\
(41,7 \%)\end{array}$ & $\begin{array}{c}84 \\
(44,2 \%)\end{array}$ \\
\hline Kassai et al. & 2018 & $\begin{array}{l}\text { São } \\
\text { Bernardo do } \\
\text { Campo, SP }\end{array}$ & $\begin{array}{l}23^{\circ} 41^{\prime} 40^{\prime \prime} \\
\text { Sul }\end{array}$ & 144 & $\begin{array}{c}50 \\
(34,5 \%)\end{array}$ & $\begin{array}{c}54 \\
(37,5 \%)\end{array}$ & $\begin{array}{c}40 \\
(34,5 \%)\end{array}$ \\
\hline TOTAL & & & & 1053 & $\begin{array}{c}336 \\
(31,9 \%)\end{array}$ & $\begin{array}{c}434 \\
(41,2 \%)\end{array}$ & $\begin{array}{c}283 \\
(26,9 \%)\end{array}$ \\
\hline
\end{tabular}

Na Tabela 1 observa-se que, quanto mais próxima está a região brasileira da linha do Equador, maior é a prevalência de gestantes com adequado estado nutricional de vitamina $\mathrm{D}$, o que denota a importância do clima e da exposição solar no estado nutricional de vitamina $\mathrm{D}$. 


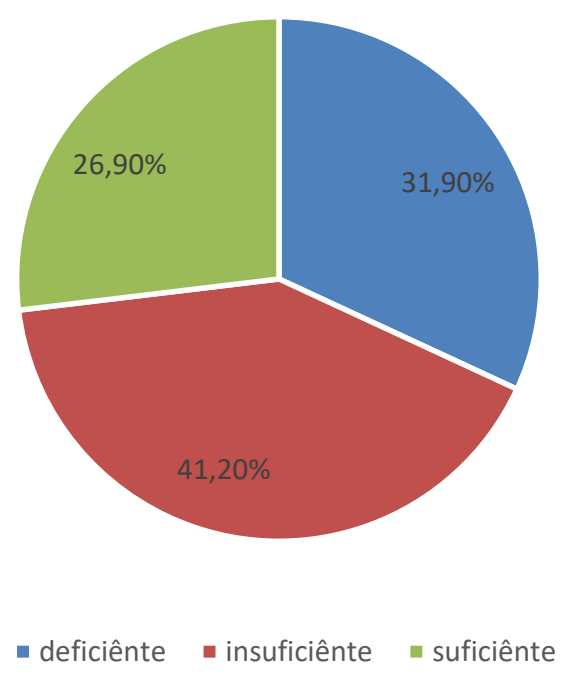

Figura 1: Distribuição do status da vitamina D em gestantes no Brasil, somando os 4 trabalhos estudados.

Ao se realizar o teste de qui-quadrado observou-se que as prevalências de estado nutricional adequado de vitamina $D$ são estatisticamente diferentes nas regiões brasileiras estudadas $(p<0,005)$.

O estudo realizado em Curitiba apresentou prevalência de adequação do estado nutricional de vitamina $D$ significativamente menor que as demais regiões. Já o estudo realizado em Santo Antônio de Jesus, apresentou prevalência de adequação do estado nutricional de vitamina $\mathrm{D}$ significativamente maior que as demais regiões.

\section{DISCUSSÃO}

A região mais próxima do Equador (Santo Antônio de Jesus- BA) apresentou maiores percentuais de adequação do estado nutricional de vitamina $D$, enquanto a mais distante (Curitiba) maior prevalência de hipovitaminose D, estatisticamente significante. Porém outros fatores devem ser considerados na avaliação do estado nutricional da vitamina D, como a etnia da população, os hábitos alimentares, a ingestão dietética da vitamina, as vestimentas típicas e a exposição solar sem o uso de protetor solar.

De forma análoga, Weller et al. (2018) estudaram gestantes da Nova Zelândia (latitude $45^{\circ}$ Sul), e encontraram $65 \%$ de deficiência de vitamina $D$, valor que supera os resultados encontrados nos estudos brasileiros analisados aqui, mas devemos destacar que a latitude mais próxima é a de Curitiba $\left(25^{\circ} 25^{\prime} 42^{\prime \prime}\right)$, onde temos $43,7 \%$ de deficiência de vitamina $D$, ressaltando a importância da distância da linha do Equador na 
Hipovitaminose D.

Em estudo realizado na Noruega foram observadas diferenças significativas nas prevalências de hipovitaminose D em diferentes etnias (EGGEMOEN et al., 2016). Nas comparações realizadas nessa revisão não foi possível considerar a influência da etnia, pois os estudos não caracterizaram as populações estudadas quanto a origem étnica, no entanto, tal comparação poderia auxiliar na compreensão dos diferentes fatores que afetam o estado nutricional da vitamina $D$.

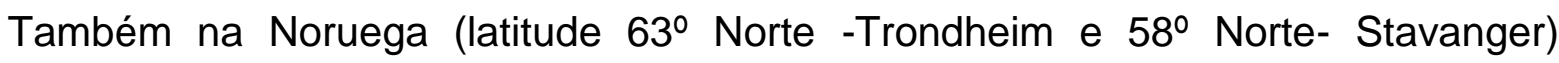
estudo de Gustafsson et al. (2018) sinalizou alta prevalência de hipovitaminose D (41\%), sendo ainda maior na estação de inverno (47\% em Trondheim e 51\% em Stavanger), condizente com menor exposição solar. Estes valores, porém são menores do que os encontrados no presente estudo $(68,1 \%)$, apesar destas cidades distarem mais do Equador que as analisadas no Brasil, o que indica que vários fatores são capazes de influenciar a dosagem da vitamina $D$, tendo destaque importante para a alimentação, visto que na Noruega tem um consumo maior de peixes que no Brasil, alimento rico em Vitamina D.

Wang et al. (2016) encontraram na China, prevalências semelhantes à média das prevalências encontradas nos 4 estudos brasileiros estudados nessa revisão: 38\% de insuficiência e $24,5 \%$ de deficiência de vitamina $D$.

Em estudo realizado por PRADO,MRMC et al., 2015, na cidade de Viçosa- MG, no Brasil, (que não entrou na meta-análise, por não ter realizado a dosagem de $25 \mathrm{OH}$ Vitamina $D$ na gestação) avaliou a hipovitaminose $D$ no puerpério imediato (valores que são semelhantes ao final da gestação), encontrando uma prevalência de $85 \%$ de hipovitaminose $D$ nestas pacientes, sendo que não ocorreu associação da dosagem de 25 $\mathrm{OH}$ vitamina $\mathrm{D}(\mathrm{p}<0,05)$ com a etnia, exposição solar, uso de protetor solar ou suplemento vitamínico. Estes valores foram próximos apenas dos encontrados em Curitiba, mesmo estando Viçosa numa latitude mais próxima do Rio de Janeiro.

\section{CONSIDERAÇOES FINAIS}

A presente revisão, apesar de contar com poucos estudos e não conseguir avaliar todas as regiões do país, mostrou uma alta prevalência de hipovitaminose D no Brasil, sendo mais expressivo quanto mais distantes da linha do Equador, porém, mesmo em 
regiões com clima equatorial ainda temos uma importante deficiência de vitamina $D$ em gestantes.

No Brasil, ainda não faz parte dos exames de rotina de pré-natal a dosagem da 25 $\mathrm{OH}$ vitamina $\mathrm{D}$ e nem a suplementação de vitamina $\mathrm{D}$ nos protocolos de assistência à gestante, e em função das complicações da hipovitaminose $D$ na gestação, faz-se necessário mudanças na política nacional de rastreamento, afim de implantar como rotina a dosagem de $250 H$ Vitamina $D$ na gestação e sua suplementação.

\section{REFERENCIAS}

ABEDI, P.; MOHAGHEGH, Z.; AFSHARY, P.; LATIFI, M. The relationship of serum vitamin D with pre-eclampsia in the Iranian women. Matern Child Nutr, 2013.

AGHAJAFARI, F et al. Association between maternal serum 25-hydroxyvitamin D level and pregnancy and neonatal outcomes: systematic review and meta-analysis of observational studies. B Met J, v.346, p.1169, 2013.

ALA-HOUHALA, M. 25- Hydroxyvitamin D levels during breast feeding with or without maternal or infantile supplementation of vitamin D. J Pediatr Gastroenterol Nutr, v.4, p.220-226, 1985.

ALOIA, J.F.; CHEN, D.G.; YEH, J.K. Serum vitamin D metabolites and intestinal calcium absorption efficiency in women. Am Am J Clin Nutr, v.92, n.4, p.835-840, 2010.

ASEMI, Z.; HASHEMI, T.; KARAMALI, M.; SAMIMI, M.; ESMAILLZADEH, A. Effects of vitamin $D$ supplementation on glucose metabolism, lipid concentrations, inflammation, and oxidative stress in gestational diabetes: a double-blind randomized controlled clinical trial. Am J Clin Nutr, 2013 Oct 16.

AUTIER, P.; GANDINI, S.; MULLIE, P. A systematic review: influence of vitamin D supplementation on serum 25-hydroxyvitamin D concentration. J. Clin Endocrinol Metab, v.97, n.8, p.606-6013, 2012.

BASILE, L. H. Gestante e a necessidade de Vitamina D. International Journal of Nutrology, v.7, n.1, p. 05-13, Jan/Abr 2014.

BARTOSZEWICZ, Z.; KONDRACKA, A.; KRASNODEBSKAKILJAŃSKA, M.; NIEDŹWIEDZKA, B.; POPOW, M.; AMBROZIAK, U.; BEDNARCZUK, T. Vitamin D insufficiency in healthy pregnant women living in Warsaw. Ginekol Pol., v. 84, n. 5, p. 362-367, 2013.

BENACHI, A.; CORDIER, A.G.; COURBEBAISSE, M.; SOUBERBIELLE, J.C. Vitamin D and pregnancy. Presse Med., v. 42, n. 10, p. 1377-1382, 2013.

BHUTTA Z.A et al. Evidence-based interventions for improvement of maternal and child nutrition: what can be done and at what cost? Lancet, v.77, p.382-452, 2013. 
BINKLEY, $\mathrm{N}$ et al. Evaluation of ergocalciferol or cholecalciferol dosing, 1,600 IU daily or 50,000 IU monthly in older adults. J Clin Endocrinol Metab, v.96, n.4, p.981-988, 2011.

CASTRO, L. C. G. O sistema endocrinológico vitamina D. Arq Bras Endocrinol Metab, v.55, n.8, 2011.

CHICOTE, C.C.; LORENCIO, F.G. Vitamina D: una perspectiva actual. Comité de Comunicación de la Sociedad Espanola de Bioquímica Clínica y Patología Molecular, Barcelona, 2013.

CHRISOSTOMO, K.R. et al. The prevalence and clinical associations of hypovitaminosis D in pregnant women from Brazil. International Journal Gynecology \& Obstetric, 2018.

DAWODU, A.; WAGNER, C. L. Prevention of vitamin D deficiency in mothers and infants worldwide - a paradigm shift. Pediatric Int Child Health, v. 3, n.2, p.3-13, 2012.

DAWODU, A.; AKINBI, H. Vitamin D nutrition in pregnancy: current opinion. Int $\mathbf{J}$ Womens Health, v.5, p.333-343, 2013.

DAWSON-HUGHES, B.; HARRIS, S.S.; DALLAL, G.E. Plasma calcidiol, sea-son, and serum parathyroid hormone concentrations in healthy elderly men and women. Am $\mathbf{J}$ Clin Nutr, v.65, p.67-71, 1997.

EGGEMOEN, A. R. et al. Vitamin D deficiency and supplementation in pregnancy in a multiethnic population- based cohort. BCM pregnancy childbirt, v.16, p.327-345, 2016.

FIGUEIREDO, A. C. C et al. Changes in plasma concentrations of 25-hydroxyvitamin D and 1,25-dihydroxyvitamin D during pregnancy: a Brazilian cohort. Eur J Nutr., v.57, n.3, p.1059-1072, 28 de março 2017.

GANNAGE-YARED, M. $\mathrm{H}$ et al. Hypovitaminosis $\mathrm{D}$ in a sunny country: relation to lifestyle and bone markers. J Bone Miner Res, v.15, n.9, p.1856-1862, 2000.

GUSTAFSSON, M.K. et al. Alterations in the vitamin D endocrine system during pregnancy: A longitudinal study of 855 healthy Norwegian women. PLoS One, 2018

HEANEY, RP. What is vitamin D insufficiency? And does it matter? Calc if Tissue Int, v.92, n.2, p.177-183, 2013.

HOLICK, M. F. Vitamin D deficiency. N Engl J Med, v.357, p.266-281, 2007.

HOLICK, M.F.; BINKLEY, N.C et al. Evaluation, treatment, and prevention of vitamin D deficiency: an Endocrine Society clinical practice guideline. J Clin Endocrinol Metab, v.96, n.7, 1911-1930, 2011.

HOLICK, M.F et al. Guidelines for preventing and treating vitamin D deficiency and insufficiency revisited. J Clin Endocrinol Metab, v.97, n.5, p.153-158, 2012. 
HOSSEIN-NEZHAD, A.; HOLICK, M.F. Vitamin D for health: a global perspective. Mayo Clin Proc, v.55, p.88-720, 2013.

INSTITUTE OF MEDICINE (IOM). Dietary Reference Intakes (DRIs) for calcium and vitamin D. Report at a glance 2011. Disponível em: http://www.iom.edu/Reports/2010/Dietary-Reference-Intakes-for- Calcium-and-VitaminD/DRI-Values.aspx.

JAIN, $V$ et al. Vita-min $D$ deficiency in healthy breasted term infants at 3 months and their mothers in India: seasonal variation \& determinants. Indian J Med Res, v.133, p.267-273, 2011.

JAMALI, Z et al. Vitamin D status in female students and its relation to calcium metabolism markers, lifestyles, and polymorphism in vitamin D receptor. Clin Lab, v.59, n.3, p.407-413, 2013.

KASSAI, M.S. Vitamin D plasma concentrations in pregnant women and their preterm newborns. BMC Pregnancy and Childbirth, v.18, n.1, p.327-345, outubro 2018.

LAI, J.K et al. Assessing vitamin D status: pitfalls for the unwary. Mol Nutr Food Res, v.54, n.8, p.1062-1071, 2010.

LIPS, P. Vitamin D deficiency and secondary hyperparathyroidism in the elderly: consequences for bone loss and fractures and therapeutic implications. Endocr Rev, v.22, n.4, p.477-501, 2001.

$M A, R$ et al. Expressions of vitamin D metabolic components VDBP, CYP2R1, CYP27B1,CYP24A1, and VDR in placents as from normal and pre-eclamptic pregnancies. Am J Physiol Endocrinol Metab, v.5, p.303-928, 2010.

MAEDA, S.S et al. Recommendations of the Brazilian Society of Endocrinology and Metabology (SBEM) for the diagnosis and treatment of hypovitaminosis D. Arq Bras Endocrinol Metab, v.58, p.411-433, 2014.

MASLOVA, E.; HANSEN, S.; JENSEN, C.B.; THORNE-LYMAN, A.L.; STRØM, M.; OLSEN, S.F. Vitamin D intake inmid-pregnancy and child allergic disease - a prospective study in 825 Danish mother-child pairs. BMC Pregnancy Childbirth, v. 13, n. 1, p. 199, 2013.

MASVIDAL, A. R. M et al. Vitamin D: pathophysiology and clinical applicability in pediatrics. An Pediatr, v.77, p.279, 2012.

MCDONNELL, D. P.; PIKE, J. W.; O'MALLEY, B. W. The vitamin D receptor: a primitive steroid receptor related to thyroid hormone receptor. J Steroid Biochem, v.30, n.1, p.41-46, 1988.

MOREIRA, $P$ et al. Treatment of vitamin D deficiency increases lower limb muscle strength in institutionalized older people independently of regular physical activity: a randomized double-blind controlled trial. Ann Nutr Metab, v.54, n.4, p.291-300, 2009. 
MOREIRA, $P$ et al. Treatment of vitamin $D$ deficiency increases lower limb muscle strength in institutionalized older people independently of regular physical activity: a randomized double-blind controlled trial. Ann Nutr Metab, v.54, n.4, p.291-300, 2009.

MULLIGAN, M. L.; FELTON, S. K.; RIEK, A. E. BERNAL-MIZRACHI, C. Implications of vitamin $D$ deficiency in pregnancy and lactation. Am J Obstet. Gynecol, v.1, n.9, p.202-429, 2010.

$\mathrm{NARCHI}, \mathrm{H}$ et al. Maternal vitamin D status throughout and after pregnancy. $\mathbf{J}$ Obstet Gynaecol, v.42, p.30-137, 2010.

NESTLE M, NESHEIM MC. To Supplement or Not to Supplement : The U . S . Preventive Services Task Force Recommendations on Calcium and Vitamin D. Ann. Intern. Med., p.701-703, 2013.

NORMAN, A.W.; BOUILLON, R. Vitamin D nutritional policy needs a vision for the future. Exp Biol Med, v.235, n.9, p.1034-1045, 2010.

POWE, C.E.; KARUMANCHI, S.A.; THADHANI, R. Vitamin D-binding protein and vitamin D in blacks and whites. N. Engl J Med, v.370, n.9, p.880-881, 2014.

PRADO, M.R.M.C et al. Prevalência de deficiência de vitamina D e fatores associados em mulheres e seus recém-nascidos no período pós-parto. Revista Paulista de Pediatria, v.33,p.286-293, 2015.

NORDIN, B. E. Evolution of the calcium paradigm: the relation between vitamin D, serum calcium and calcium absorption. Nutrients, v.2, n.9, p.997-1004, 2010.

PEREIRA-SANTOS, M. Vitamin D deficiency and associated factors among pregnant women of a sunny city in Northeast of Brazil. Clinical Nutrition ESPEN, v.23, p.240244, Fevereiro 2018.

PETERS, B.S.E et al. Prevalence of vitamin D insufficiency in Brazilian adolescents. Ann Nutr Metab, v.54, n.1, p.15-21, 2009.

PINHEIRO, M. M et al. Nutrient intakes related to osteoporotic fractures in men and women- the Brazilian Osteoporosis Study (BRAZOS). Nutr J, v.8, n.6, 2009.

REICHRATCH, J.; NURNBERG, B. Cutaneous vitamin D synthesis versus skin cancer development: The Janus faces of solar UV-radiation. Rev Dermato endocrinol, v.1, n.5, p.253-261, 2009.

ROSS, A. C et al. The 2011 report on dietary reference intakes for calcium and vitamin $\mathrm{D}$ from the Institute of Medicine: what clinicians need to know. $\mathbf{J}$ Clin Endocrinol Metab, v.96, n.1, p.53-58, 2011.

SCHUCH, N. J.; GARCIA, V. C. Vitamina D e doenças endocrinometabólicas. Arq Bras Endocrinol Metab, p.625-633, 2009.

WANG, C. et al. Correlation between neonatal vitamin D level and maternal vitamin D level. Zonagguo Dang Dai Er Ke Za Zhi, 2016 